\title{
A comparison of temperament and character among psychiatry, internal medicine, and surgery residents at Iran University of Medical Sciences in the academic year 2013-2014
}

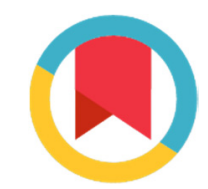

\author{
Nasibeh Hashemkhani ${ }^{1}$, Ruohollah Seddigh ${ }^{2}$, Amir-Abbas Keshavarz-Akhlaghi ${ }^{2}$, Ahmad Mousavi ${ }^{1}$, Shiva Soraya*2
}

Received: 27 Jan 2018

Published: 13 Nov 2019

\begin{abstract}
Background: This study was conducted to compare temperament and character among psychiatry residents, internal medicine, and surgery residents in hospitals affiliated to Iran University of Medical Sciences in the academic year 2013-2014.

Methods: This cross sectional analytical study was conducted on 201 residents using the non-probability method of convenience sampling. Cloninger's 125-item Temperament and Character Inventory (TCI) and Maslach's Burnout Inventory (MBI) were distributed among all the residents in their morning session. Once the questionnaires were completed and submitted, the data were analyzed in SPSS 16. The frequency of different personality traits was analyzed among the selected residents.

Results: A total of $49.8 \%$ of the respondents were female $(n=100)$ and $50.2 \%$ were male $(n=101)$. The results of data analysis showed more novelty seeking, reward dependence, and harm avoidance and less persistence $(33.93, \mathrm{p}=0.006 ; 9.00, \mathrm{p}=0.056 ; 32.55$, $\mathrm{p}=0.021$; and $2.48, \mathrm{p}=0.028$, respectively) in psychiatry residents than in surgery residents $(31.97,7.87,30.74$, and 3.12 respectively). Reward dependence was more frequent in internal medicine residents than in surgery residents $(9.44$ and 7.87 respectively, $\mathrm{p}=0.002)$. The self-directedness score was lower in psychiatry residents than in internal medicine residents $(\mathrm{p}=0.761)$ and higher than in surgery residents $(17.96,18.30$, and 17.57 respectively, $\mathrm{p}=0.824)$. The cooperativeness and self-transcendence scores were higher in psychiatry residents than in internal medicine ( $\mathrm{p}=0.943, \mathrm{p}=0.199$, respectively) and surgery residents $(\mathrm{p}=0.105, \mathrm{p}=0.069$ respectively).

Conclusion: The different dimensions of personality varied between the surgery, internal medicine, and psychiatry residents. Particularly, this study did not show statistically significant differences in dimensions of character (cooperativeness, self-directedness, and self-transcendence) among surgery, internal medicine, and psychiatry residents. The selection of medical specialties based on character traits significantly contributes to more effective treatment of patients and higher satisfaction of the residents.
\end{abstract}

Keywords: Character, Medical resident, Psychiatrist, Internist, Surgeon

Conflicts of Interest: None declared

Funding: None

*This work has been published under CC BY-NC-SA 1.0 license.

Copyright $₫$ Iran University of Medical Sciences

Cite this article as: Hashemkhani N, Seddigh R, Keshavarz-Akhlaghi AA, Mousavi A, Soraya Sh. A comparison of temperament and character among psychiatry, internal medicine, and surgery residents at Iran University of Medical Sciences in the academic year 2013-2014. Med J Islam Repub Iran. 2019 (13 Nov);33:121. https://doi.org/10.47176/mjiri.33.121

Introduction

Character represents all the unique ways that a person chooses to adapt to the changes in their inside and outside

Corresponding author: Dr Shiva Soraya, soraya.s@iums.ac.ir

1. School of Medicine, Iran University of Medical Sciences, Tehran, Iran

2. Spiritual Health Research Center, Iran University of Medical Sciences,

Tehran, Iran $\uparrow$ What is "already known" in this topic:

Personality traits reflect the individual's mental abilities/efficiency, which should be addressed when guiding for career choices and field of study. Previous studies have shown that those with high IQs are not suitable for certain fields of study or work, because they lack certain personality traits and can instead achieve significant success in other academic/ career environments.

$\rightarrow$ What this article adds:

Given the lack of similar studies and the need for research in this field, especially in Iran, and since the results of this study may help choose a medical specialty in accordance with personal characteristics, the present study examined and compared the frequency of different character traits in medical residents of different specialties. 
world and determines the individual's lifestyle. Choosing a career can also be a function of the dimensions of one's character (1). Personality traits reflect the individual's mental abilities and efficiency and should be considered when choosing a career or a field of study (2). Personality traits have a direct relationship with the degree of burnout in medical students and residents $(3,4)$. A study among health care staff based on the Cloninger questionnaire and Mazlach's questionnaire, showed that personality traits can predict the incidence of burnout (5). Studies have revealed that some people with high IQs are not suitable for certain fields of study or work, because they lack certain personality traits, but they can instead achieve significant success in other academic and career environments. In other words, people's personality traits make them appropriate for specific fields of work (6). The field of study or work should provide a suitable context for the individual to meet their personal and emotional needs, and since different disciplines and professions are set within different emotional and intellectual contexts, personal differences inevitably play a role in the type of occupation chosen (7). Developed countries currently place great emphasis on giving personality tests to candidates to help them choose a more suitable field of study or work (8). Studies also showed that some traits are more dominant among students of certain disciplines. For example, surgery residents have a greater conscientiousness and more extraversion and emotional stability compared to the general population, however, residents of other disciplines and first-year medical students have less openness compared to the general population (9). Compared to the general population, internal medicine residents are more caring toward others, have a deeper intellectual curiosity, greater idealism and clearer perceptions, and are more acceptable of their own feelings and think more carefully before they act. Compared to those in other specialty fields, psychiatry residents have more positive mental health and more recognizable character traits. Cloninger et al introduced the psychobiological theory in 1993 and attributed 4 dimensions to temperament and 3 to character. The 4 dimensions of temperament are as follow: (1) novelty seeking, (2) harm avoidance, (3) reward dependence, and (4) persistence. Also, the 3 dimensions of character are as follow: (1) self-directedness, (2) cooperativeness, and (3) self-transcendence. Cloninger believes that character is less inherited than temperament and that it matures with age (10). The results of a broad survey of personality traits conducted at Mazandaran University of Medical Sciences in 2011 based on the Big Five personality traits showed that the different traits assessed were more dominant in one of the faculties; that is, a high percentage of the students of a given faculty had homogeneous characteristics (11). Studies are indicative of differences between the character of surgery residents and the character of residents in other disciplines. In a study conducted to compare surgery residents with the general population and firstyear medical students based on the Big Five personality traits, surgery residents obtained higher scores in conscientiousness, extraversion, and emotional stability, while they were less willing to experience new situations, eg, had a lower openness $(9,12)$. There is a lack of similar studies and a need for research in this field, especially in Iran. The results of this study may be useful in helping to choose a medical specialty in accordance with personal characteristics. Moreover, the study results can be used to increase the awareness of residents about the characteristics of their personality and also help teachers consider the personality features of students in their educational guidance. The present study was conducted to examine and compare the frequency of different character traits in medical residents of different specialties.

\section{Methods}

This cross sectional analytical study was conducted using the nonprobability method of convenience sampling. The study population consisted of 201 residents in the first, second, third, and fourth years of psychiatry, internal medicine, and surgery at hospitals affiliated to Iran University Medical Sciences in the academic year 2013-2014. The participants were medical graduates who had entered a specialized residency program based on their personal interest and the scores they obtained in the national university entrance examination for medical residency. A copy of the questionnaire was obtained from the Mental Health Research Center and the School of Behavioral Sciences and Mental Health; arrangements were made with the heads of the 3 departments to distribute the questionnaires among all the residents in the different years of their field in their morning meeting to be filled and returned in the same meeting. If any of the residents could not return their completed questionnaire in the same meeting, the researcher collected them in the following day. Lack of cooperation and partial completion of the questionnaires was approximately $16 \%$. The questionnaires inquired about the respondents' demographic information and contained Cloninger's 125-item Temperament and Character Inventory (TCI) and Maslach's Burnout Inventory (MBI). Once the questionnaires were completed and submitted, the frequency of the different personality traits was analyzed in different residency disciplines and its relationship with job satisfaction and burnout was also evaluated and reported in a separate paper (13). These 3 residency fields were selected because of their nearly similar minimum scores accepting residents and their differences in content and practical applications.

The researchers fully adhered to all the principles of the Declaration of Helsinki (eg, the ethical principles for medical research involving humans) and the ethics rules were approved by the Ministry of Health.

Statistical analyses were performed using SPSS 16 (IBM; Chicago, IL, USA). Also, one-sample KS was used to test the normality of distribution of continuous data. Mean \pm SD was reported for normally distributed variables and median (IQR) for data without normal distribution.

Non-paired t tests and one-way ANOVA with post hoc analysis (Tukey's test) were used to compare parametric continuous variables (TCI score) among 2 and more than 2 groups, respectively. Parametric tests were also used to assess Likert scales (MBI score A 2-sided $p$ value $<0.05$ was considered statistically significant). 


\section{Results}

A total of $49.8 \%$ of the respondents were female $(\mathrm{n}=100)$ and $50.2 \%$ were male $(\mathrm{n}=101)$. The median age of the participants was 31 (IQR: 5). Also, 35.8\% $(n=72)$ were internal medicine residents, $35.3 \%(\mathrm{n}=71)$ surgery residents, and $28.9 \%(\mathrm{n}=58)$ psychiatry residents. Moreover, $45.8 \%$ of the participants $(n=92)$ were single and $54.2 \%(n=109)$ were married. Table 1 presents the mean scores obtained in the various subgroups of the TCI. The results of the analysis using Tukey's post hoc test showed that the mean difference of novelty seeking was 1.12 (SE: 0.62 ), which was 1.95 (SE: 0.62) units higher in psychiatry residents than in internal medicine and surgery residents $(\mathrm{p}=0.174 ; \mathrm{p}=0.006)$, suggesting a statistically significant difference. This score was 0.83 (SE: 0.59) units lower in surgery residents than in internal medicine residents $(p=0.342)$. Table 2 compares the groups in the TCI. The results of the analysis suggest a statistically significant difference (Table 2).

\section{Discussion}

This study examined the personality traits of a number of medical residents in 3 fields: psychiatry, internal medicine, and surgery. The results showed that psychiatry residents were more novelty-seeking and harm-avoidant and less persistent than surgery residents. Reward dependence was higher in internal medicine residents than in surgery residents. In a study by Barbara Buddeberg-Fischer et al, the frequency of the 2 genders was nearly equal in the field of internal medicine. In surgery, the frequency of the male students was about 5 times higher than that of female students. In psychiatry, the frequency of female students was slightly higher than that of male students (14). In a study by Bradley et al, the frequency of male and female students was almost equal in internal medicine and psychiatry, while the frequency of the male students was 2 times higher than that of the female students in the field of surgery (15).

Overall, the median of percentage of interest in the field of study was $80 \%$. The score of novelty- seeking was 1.95 units higher in psychiatry residents than in surgery residents, suggesting a statistically significant difference. The higher novelty-seeking in these residents may be due to the nature of psychiatry. In a study by Barbara Buddeberg-Fischer et al, the mean score of novelty-seeking was $9.2 \pm 2.7$ in surgeons (14), but in the present study, this score was much higher in surgery residents yet lower in the residents of the other 2 disciplines. Furthermore, the score of harm avoidance was 1.80 units higher in psychiatry residents than in surgery residents, suggesting a statistically significant difference. The higher harm avoidance in psychiatry residents can be justified by noting that these students choose to study in a field that has fewer shifts just to be on the safe side, while surgery residents, who were found to have a lower harm avoidance, enter a field with higher workload and more frequent shifts. The anxiety of entering a field with greater responsibilities, one that requires decision-making on the spot, and the dominance of females among psychiatry residents can be other reasons. Nonetheless, these assumptions need to be further studied

\begin{tabular}{|c|c|c|c|c|c|}
\hline \multicolumn{2}{|l|}{ Variable } & Internal medicine residents & Surgery residents & \multicolumn{2}{|c|}{ Psychiatry resident } \\
\hline \multirow{3}{*}{ Temperament } & & \multicolumn{4}{|c|}{ Mean \pm SD } \\
\hline & Novelty seeking & $32.80 \pm 2.64$ & $31.97 \pm 2.27$ & \multicolumn{2}{|c|}{$33.93 \pm 5.37$} \\
\hline & Harm avoidance & $31.80 \pm 3.16$ & $30.74 \pm 3.15$ & \multicolumn{2}{|c|}{$32.55 \pm 5.05$} \\
\hline \multirow{5}{*}{ Character } & Reward dependence & $9.44 \pm 2.06$ & $7.87 \pm 3.46$ & \multicolumn{2}{|c|}{$9.00 \pm 2.48$} \\
\hline & Persistence & $2.91 \pm 1.09$ & $3.12 \pm 1.57$ & \multicolumn{2}{|c|}{$2.48 \pm 1.53$} \\
\hline & Self-directedness & $18.30 \pm 3.63$ & $17.57 \pm 3.19$ & \multicolumn{2}{|c|}{$17.96 \pm 4.29$} \\
\hline & Cooperativeness & $20.97 \pm 2.31$ & $19.73 \pm 5.80$ & \multicolumn{2}{|c|}{$21.20 \pm 3.10$} \\
\hline & Self-transcendence & $7.00 \pm 3.66$ & $6.59 \pm 4.06$ & \multicolumn{2}{|c|}{$8.37 \pm 5.86$} \\
\hline Indicator & Group I & Group J & Mean difference $(\mathrm{I}-\mathrm{J})$ & Std. error & $\mathrm{p}$ \\
\hline \multirow[t]{3}{*}{ Novelty seeking } & Psychiatry resident & Internal medicine resident & 1.12 & 0.62 & 0.174 \\
\hline & Surgery resident & Surgery resident & 1.95 & 0.62 & 0.006 \\
\hline & & Internal medicine resident & -0.83 & 0.59 & 0.342 \\
\hline \multirow[t]{3}{*}{ Harm avoidance } & Psychiatry resident & Internal medicine resident & 0.74 & 0.67 & 0.507 \\
\hline & Surgery resident & Surgery resident & 1.80 & 0.62 & 0.021 \\
\hline & & Internal medicine resident & -1.05 & 0.63 & 0.221 \\
\hline \multirow[t]{3}{*}{ Reward dependence } & Psychiatry resident & Internal medicine resident & -0.44 & 0.48 & 0.630 \\
\hline & Surgery resident & Surgery resident & 1.12 & 0.48 & 0.056 \\
\hline & & Internal medicine resident & -1.57 & 0.45 & 0.002 \\
\hline \multirow[t]{3}{*}{ Persistence } & Psychiatry resident & Internal medicine resident & -0.43 & 0.24 & 0.192 \\
\hline & Surgery resident & Surgery resident & -0.64 & 0.24 & 0.028 \\
\hline & & Internal medicine resident & 0.21 & 0.23 & 0.647 \\
\hline \multirow[t]{3}{*}{ Self-directedness } & Psychiatry resident & Internal medicine resident & -0.34 & 0.65 & 0.861 \\
\hline & Surgery resident & Surgery resident & 0.38 & 0.65 & 0.824 \\
\hline & & Internal medicine resident & -0.72 & 0.61 & 0.468 \\
\hline \multirow[t]{3}{*}{ Cooperativeness } & Psychiatry resident & Internal medicine resident & 0.23 & 0.71 & 0.943 \\
\hline & Surgery resident & Surgery resident & 1.47 & 0.72 & 0.105 \\
\hline & & Internal medicine resident & -1.23 & 0.68 & 0.166 \\
\hline \multirow[t]{3}{*}{ Self-transcendence } & Psychiatry resident & Internal medicine resident & 1.37 & 0.80 & 0.199 \\
\hline & Surgery resident & Surgery resident & 1.78 & 0.80 & 0.069 \\
\hline & & Internal medicine resident & -0.40 & 0.75 & 0.852 \\
\hline
\end{tabular}


to ensure their accuracy. Buddeberg-Fischer et al found the mean score of harm avoidance to be $8.1 \pm 5.1$ in surgeons, which is much lower than the score obtained in the present study (14), in which surgery residents received the lowest harm avoidance score of all the 3 groups. The score of reward dependence was 1:57 units lower in surgery residents than in internal medicine residents, suggesting a statistically significant difference. Buddeberg-Fischer et al found the mean score of reward dependence to be $8.0 \pm 2.7$ in surgeons, which is almost equal to the score obtained in the present study (14). The lower score of reward dependence in surgery residents may be due to how people with higher reward dependence are more sensitive to verbal reward and earning the community's approval, and since in surgery, interaction with the patients is less than in the fields of internal medicine and psychiatry, surgery residents are often among those with a lower reward dependence. In internal medicine, the physician is more capable of improving or controlling the disease, which explains why internal medicine residents received the highest reward dependence score. In this study, the score of persistence was 0.64 units lower in psychiatry residents than in surgery residents, suggesting a statistically significant difference. Buddeberg-Fischer et al found the mean score of persistence to be $3.1 \pm 105$ in surgeons, which is similar to the score obtained in the present study (14). The higher score of persistence in internal medicine residents in this study can be due to the fact that internal medicine requires ongoing education during the prolific and difficult time of residency, and students often decide on continuing their education up to receiving specialty degrees before they enter this field. As a result, students who choose this field require more persistence. The mean score of the selfdirectedness subgroup of the TCI was $18.30 \pm 3.63$ in internal medicine residents, $17.96 \pm 4.29$ in psychiatry residents, and $17.57 \pm 3.19$ in surgery residents, suggesting the lack of a statistically significant difference. BuddebergFischer et al found the mean score of self-directedness to be $15.9 \pm 4.1$ in surgeons, which is consistent with the score obtained in the present study (14). The mean score of the cooperativeness subgroup of the TCI was $21.20 \pm 3.10$ in psychiatry residents, $20.97 \pm 2.31$ in internal medicine residents, and $19.73 \pm 5.80$ in surgery residents, suggesting the lack of a statistically significant difference. Buddeberg-Fischer et al found the mean score of cooperativeness to be $16.5 \pm 4.2$ in surgeons, which is lower than the score obtained in the present study. The mean score of the self-transcendence subgroup of the TCI was $8.37 \pm 5.86$ in psychiatry residents, $7.00 \pm 3.66$ in internal medicine residents, and $6.59 \pm 4.06$ in surgery residents, suggesting the lack of a statistically significant difference. Buddeberg-Fischer et al found the mean score of selftranscendence to be $6.7 \pm 3.3$ in surgeons, which is consistent with the score obtained in the present study (14). The findings of the present study are consistent with other studies in the field that have used personality theories and other measuring tools in the field of personality of various physicians (16-18).

The limitations of the study include the lack of cooperation on the part of the residents, which was obviated after they were briefed on the objectives and significance of the study, and also the incomplete questionnaires (The estimated response rate was about $80 \%$, which was considered in determining the sample size). Nevertheless, some personality traits were extracted from some of the incomplete questionnaires. Also, those questionnaires in which only a single choice was selected in response to all the items and questionnaires that followed a devised pattern of responding were excluded from the study.

\section{Conclusion}

The different dimensions of personality differed between the surgery, internal medicine, and psychiatry residents. Particularly, this study did not show statistically significant differences in dimensions of character (cooperativeness, self-directedness, and self-transcendence) between surgery, internal medicine, and psychiatry residents. This lack of difference may mean that not all physicians choose their specialties based on their personality traits. Many other factors are also involved in this selection, such as considerations for future economic conditions in the field, preferring a high quality of life over other things, the social status of the field, and the field being compatible with the individual's cultural conditions, the community and sometimes gender. However, when there is more compatibility between the individual's personality traits and their selected specialty, work efficiency and job satisfaction increase enormously and the probability of job burnout also decreases.

\section{Conflict of Interests}

The authors declare that they have no competing interests.

\section{References}

1. Cloninger CR, Svarakic DM. Personality disorders. In: Sadock BJ, Sadock VA, Ruiz P, editors. Comprehensive textbook of psychiatry. 10th ed. Baltimore: Williams \& Wilkins; 2017.

2. Quintero AJ, Segal LS. The personal interview: Assessing the potential for personality similarity to bias the selection of orthopaedic residents. Acad Med. 2010;84(10):1364-72.

3. Soo JL, Young JC, Han C. The effects of personality traits on academic burnout in Korean medical students. Integ Med Res. 2017;6(2): 207-13

4. Ryoei M, Hisae M, Ryuichiro T, Hiroyuki K, Hiroshi A, Yasushi I. Burnout in Japanese residents and its associations with temperament and character. Asian J Psych. 2016;24:5-9.

5. Devebakan N, Dogan O, Ceylan V, Akin E, Kose S. Relationship between temperament and character dimensions of personality and burnout and management in healthcare organization workers. Psych Clin Psychopharmacol. 2017;28:73-9.

6. Hakimi S, Hejazi E, Gholamali Lavasani M. The relationships between personality traits and students' academic achievement. Procedia Soc Behav Sci. 2011;29:836-45.

7. Blum ML, Naylor JC. Industrial psychology: Its theoretical and social foundations. Delhi: Co-CBS; 2004.

8. Ghorbani A, Noroozi K, Jahani-Hashem H. Correlation between type of personality and academic achievement of nursing students in the University of Medical Sciences Qazvin. Int J Nurs Res. 2013;8(1):2431.

9. Hoffman BM, Coons MJ, Kuo PC. Personality differences between surgery residents, nonsurgery residents, and medical students. Surg. 2010;148(2):187-93.

10. Sievert M, Zwir I, Cloninger KM, Lester N, Rozsa S, Cloninger CR. The influence of temperament and character profiles on specialty choice and well-being in medical residents. Peer J. 2016;6. 
11. Magalhães E, Costa P, Costa MJ. Empathy of medical students and personality: Evidence from the Five-Factor Model. Med Teach. 2012;34(10):807-12.

12. Abedi G, Mohammadi A, Mohammadi F, Alizadeh A, Hosseini H, Rostami F. University students' personality profile based on Casta \& MaCrea Five factor theory. Int J Collabor Res Inter Med Pub Health. 2012;4(6):1330-36.

13. Haghshenas M, Seddigh RA, Hashemkhani N, Keshavarz AA, Mousavi A, Soraya SH. Comparison of occupational burnout scores among medical residents and their correlation with dimensions of TCI questionnaire. Med J Islam Repub Iran. 2019(6 Nov);33:118.

14. Buddeberg-Fischer B, Klaghofer R, Abel T, Buddeberg C. Swiss residents' speciality choices - impact of gender, personality traits, career motivation and life goals. BMC Health Serv Res. 2006;6:137.

15. Maron, BA, Fein S, Maron BJ, Hillel AT, El Baghdadi MM, Rodenhauser P. Ability of prospective assessment of personality profiles to predict the practice specialty of medical students. Proc (Bayl Uni Med Cent). 2007;20:22-6.

16. Mehmood SI, Kumar A, Al-Binali A, Borleffs JC. Specialty preferences: Trends and perceptions among Saudi undergraduate medical students. Med Teach. 2012;34:S51-S60.

17. Al-Alawi M, Al-Sinawi H, Al-Husseini S, Al-Adawi S, Panchatcharam SM, Khan S, Jeyaseelan L. Influence of Eysenckian personality traits in choice of specialization by young Omani doctors. Oman Med J. 2017;32:291.

18. Jordan J, Linden JA, Maculatis MC, Hern Jr HG, Schneider JI, Wills CP, Yarris LM. Identifying the emergency medicine personality: A multisite exploratory pilot study. AEM Educ Train. 2018;2:919. 